\title{
Tracheoesophageal fistula
}

\author{
Joye Varghese • Vallath Balakrishnan • \\ Venkadeswaran Narayanan
}

Received: 31 August 2009 / Accepted: 11 January 2010 /Published online: 11 May 2010

(C) Springer-Verlag London Ltd 2010

A 26-year-old lady was referred for evaluation of dysphagia. The patient had consumed $50 \mathrm{ml}$ of organophosphorus (OP) components of liquid pesticide 4 months ago and was on ventilatory support with a tracheostomy tube because of OP-poisoning-induced respiratory failure. Treatment included injection of atropine, pralidoxime and other supportive care, and the patient recovered. The patient developed dysphagia after extubation of the tracheostomy tube, and a percutaneous endoscopic gastrostomy (PEG) feeding tube was placed to support her nutritional status. Clinically, there was no neurological deficit. Figure 1 shows the esophagogastroduodenoscopy (OGD). What is the diagnosis?

OP components are esters, amides or thiol derivatives of phosphoric, phosphonic or phosphinic acids [1]; by inhibiting acetylcholinestrase, excessive acetylcholine at the cholenergic synaptic level produces OP poisoning. TEF is an abnormal communication between the trachea and esophagus. Esophageal malignancy is the most common cause of TEF in adults; other benign causes are induced by endotracheal tubes, tracheostomies and thoracic surgery; by caustic injuries and esophageal infections like tuberculosis, syphilis and histoplasmocytosis. Ono's sign [2], i.e., cough while swallowing, is an important indication of tracheal/bronchial esophageal fistula. In our case, bronchoscopy revealed TEF at the 7 o'clock position, $5 \mathrm{~cm}$ from

J. Varghese $(\bowtie) \cdot$ V. Balakrishnan $\cdot$ V. Narayanan

Department of Gastroenterology,

Amrita Institute of Medical Sciences \& Research Centre, Cochin, India 682026

e-mail: joyevarghese@aims.amrita.edu

V. Balakrishnan

e-mail: vallathbalakrishnan@aims.amrita.edu

V. Narayanan

e-mail: vanarayanan@aims.amrita.edu

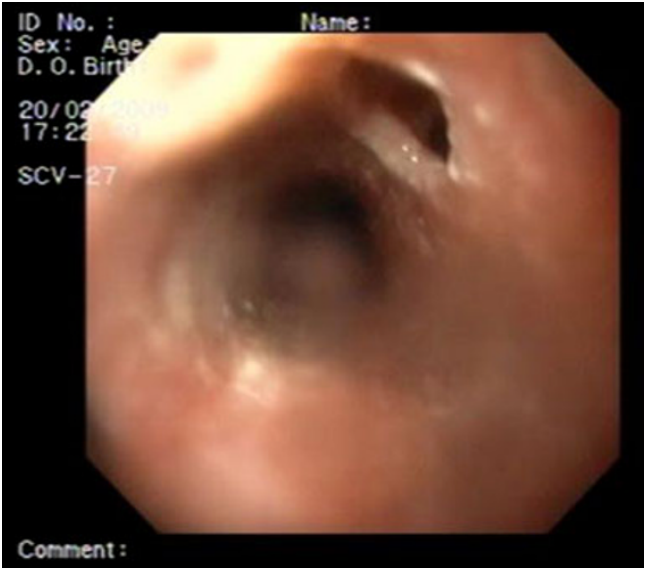

Fig. 1

the vocal cord at the junction of the cartlaginous and membranous portion, which corresponded to the lower end of the tracheostomy scar. The incidence of TEF following tracheostomy is less than $1 \%$ [3]. To our knowledge, this is the first case report of tracheostomy-induced TEF in a patient with organophosphorus poisoning. Our patient underwent tracheo-esophageal fistulectomy and closure with a free sternocleidomastoid interposition muscle flap. The PEG tube was removed 1 month after surgery.

\section{References}

1. Balali-Mood M, Kia Balali-mood (2008) Neurotoxic disorders of organophosphorus compounds and their managements. Arch Iranian Med 11(1):65-89

2. Gerzic Z, Rakic S, Randjelovic T (1990) Acquired benign esophagorespiratory fistula: report of 16 consecutive cases. Ann Thorac Surg 50:724-727

3. Reed MF, Mathisen DJ (2003) Tracheoesophageal fistula. Chest Surg Clin North Am 13(2):271-289 